\title{
Nonsinusoidal current and current reversals in a gating ratchet
}

\author{
Luis Dinis ${ }^{1,2}$ and Niurka R. Quintero ${ }^{3,4}$ \\ ${ }^{1}$ Departamento de Física Atómica, Molecular y Nuclear, Universidad Complutense de Madrid, 28040 Madrid, Spain \\ ${ }^{2}$ GISC-Grupo Interdisciplinar de Sistemas Complejos, Madrid, Spain \\ ${ }^{3}$ Instituto de Matemáticas de la Universidad de Sevilla (IMUS) \\ ${ }^{4}$ Departamento de Física Aplicada I, E.P.S., Universidad de Sevilla, Calle Virgen de África 7, 41011 Sevilla, Spain
}

(Received 4 September 2014; published 23 March 2015)

\begin{abstract}
In this work, the ratchet dynamics of Brownian particles driven by an external sinusoidal (harmonic) force is investigated. The gating ratchet effect is observed when another harmonic is used to modulate the spatially symmetric potential in which the particles move. For small amplitudes of the harmonics, it is shown that the current (average velocity) of particles exhibits a sinusoidal shape as a function of a precise combination of the phases of both harmonics. By increasing the amplitudes of the harmonics beyond the small-limit regime, departures from the sinusoidal behavior are observed and current reversals can also be induced. These current reversals persist even for the overdamped dynamics of the particles.
\end{abstract}

DOI: 10.1103/PhysRevE.91.032920

PACS number(s): 05.45.-a, 05.40.-a, 05.60.-k

\section{INTRODUCTION}

The transport of particles or solitons under zero-average forces (i.e., ratchet transport) has been extensively investigated in the last two decades [1-5]. This phenomenon has been predicted and explained in different fields of physics, ranging from nanodevices to molecular motors [4-6]. Moreover, it has also been observed in experiments and simulations with nonlinear systems, where spatiotemporal symmetries have been properly broken [7-12]. In particular, the ratchet models were used to elucidate the working principles of molecular motors; to design molecular motors [13] and to explain the unidirectional motion of fluxons in Josephson junctions [12,14], the transport of cold atoms in optical lattices [15], and the vortices in superconductors $[9,16]$.

The ratchet transport is described by means of the current (average velocity) [2,4,5,17],

$$
v=\lim _{t \rightarrow \infty} \frac{\langle x(t)\rangle-x_{0}}{t-t_{0}},
$$

where $x(t)$ is the position of particles, or the center of mass of solitons at time $t,\langle\cdot\rangle$ represents an ensemble average over all trajectories satisfying the same initial condition, and $x\left(t_{0}\right)=$ $x_{0}$.

Two possible underlying mechanisms of rocking ratchets are harmonic mixing and gating. The current of particles (atoms or solitons) in harmonic mixing is generally induced by an additive biharmonic, $T$-periodic, driving force $f(t)=$ $f_{1}(t)+f_{2}(t)$, with

$$
f_{1}(t)=\epsilon_{1} \cos \left(q_{1} \omega t\right), \quad f_{2}(t)=\epsilon_{2} \cos \left(q_{2} \omega t+\phi\right),
$$

where $\epsilon_{1}$ and $\epsilon_{2}$ are the amplitudes of the harmonics, $\phi$ is the relative phase between the two harmonics, $\left(q_{1}, q_{2}\right) \in \mathbb{N}^{2}$, $\operatorname{gcd}\left(q_{1}, q_{2}\right)=1$, and $T=2 \pi / \omega$. On the other hand, in gating ratchets, particles experience a symmetric potential with the amplitude modulated by means of $f_{1}(t)$. A time-symmetric harmonic force $f_{2}(t)$ is also applied.

The time-shift invariance of the current,

$$
v\left[f_{1}(t+\tau), f_{2}(t+\tau)\right]=v\left[f_{1}(t), f_{2}(t)\right],
$$

$\forall \tau$, together with the symmetry

$$
v\left[-f_{1}(t),-f_{2}(t)\right]=-v\left[f_{1}(t), f_{2}(t)\right],
$$

or

$$
v\left[f_{1}(t),-f_{2}(t)\right]=-v\left[f_{1}(t), f_{2}(t)\right],
$$

fix the necessary conditions on $q_{1}$ and $q_{2}$ in Eq. (2) to obtain the ratchet effect in harmonic mixing and gating. Symmetry (4) holds for rocking ratchets induced by an additive biharmonic force, whereas (5) characterizes the gating average velocity. When $q_{1}+q_{2}$ is an odd integer number, the biharmonic force $f(t)$ breaks the time-shift symmetry $f(t)=-f(t+T / 2)$ and a current appears. In a gating ratchet, if $q_{1}$ is an odd integer number, $f_{1}(t)$ preserves the time-shift symmetry, where $f_{1}(t)=-f_{1}(t+T / 2)$. Nevertheless, the gating effect appears due to a synchronization of the oscillations of the potential barrier caused by a single harmonic $f_{1}(t)$ with the motion produced by the additive harmonic force, $f_{2}(t)$. There is no constraint on $q_{2}$ in gating, and therefore a current can be obtained even for $q_{1}=q_{2}=1[18,19]$.

Moreover, Eqs. (3)-(5) together with the functional representation of the ratchet velocity determine the dependence of the current on the amplitudes and relative phase of the harmonics [20,21]. For instance, for the small-amplitude limit of the biharmonic force $f(t)=f_{1}(t)+f_{2}(t)$ with (2), the current reads

$$
v[f(t)]=A_{0} \epsilon_{1}^{q_{2}} \epsilon_{2}^{q_{1}} \cos \left(q_{1} \phi+\theta_{0}\right),
$$

where $q_{1}+q_{2}$ is an odd integer number. Otherwise the current $v$ vanishes $[20,21]$. The constants $A_{0}$ and $\theta_{0}$ are determined by the other parameters of the system (potential, dissipation, etc.). Equation (6) clearly shows the harmonic mixing since the parameters of the first harmonic always appear in combination with the parameters of the second harmonic. Interestingly, for a gating ratchet, it is deduced (for a small-amplitude limit) that $v$ again is ruled by Eq. (6), however only $q_{1}$ should be an odd integer number, whereas $q_{2}$ can be either an odd or even integer number. This formula predicts a sinusoidal dependence of $v$ versus the phase $\phi$. This implies, for example, that current reversals can be induced by solely changing the relative phase between $f_{1}$ and $f_{2}$. Furthermore, in Ref. [21], for a nonsmall 
amplitude limit, two interesting effects have been theoretically predicted: a deviation from the sinusoidal shape of $v$ as a function of the phase; and the dependence of $\theta_{0}$ and $A_{0}$ on the amplitudes of the forces. This latter fact leads to an unexpected phenomenon related with the appearance of current reversals by changing the amplitudes of the harmonics. This explains the experiments in optical lattices driven by a biharmonic force reported in Ref. [22], and in a shaken liquid drop driven by two independent harmonics [23].

In this work, we focus on the ratchet dynamics of Brownian particles lying in a symmetric potential, modulated by a harmonic function. The particles are driven by an external sinusoidal (harmonic) force. We show that there is a deviation from the sinusoidal behavior of $v$ as a function of the relative phase between the two harmonics in the nonsmall amplitude limit. Moreover, the current reversals by means of increasing the amplitudes of the harmonics are shown.

The paper is organized as follows. In the next section, the symmetry properties of the Langevin equation and its relation with the functional representation of the current predicted in Ref. [21] are described. In Sec. III, the analytical predictions of the previous section are verified by means of simulations. In addition to a class of current reversals, determined by dissipation-induced symmetry breaking [22], we show that the current reversals persist even for the overdamped dynamics of our model. To conclude the paper, in the final section, the results of Secs. II-III are discussed, thereby making the connection with the experiments and summarizing our main findings.

\section{GATING RATCHET MODEL}

In our theoretical analysis, the dynamics of particles in the spatially symmetric potential is determined by the Langevin equation

$$
m \ddot{x}=-\alpha \dot{x}-U^{\prime}(x)\left[1+f_{1}(t)\right]+f_{2}(t)+\sqrt{2 D} \xi(t),
$$

where $m$ is the mass; $U(x)=U_{0} \cos (x)$ is a periodic symmetric potential, modulated by the harmonic $f_{1}(t)$ given by $(2), \alpha$ the friction coefficient, $\xi(t)$ a Gaussian white noise, $\langle\xi(t)\rangle=0$, $\left\langle\xi(t) \xi\left(t^{\prime}\right)\right\rangle=\delta\left(t-t^{\prime}\right)$. Generally, noise smooths the dependence of the current on the parameters of the harmonics [24]. In some cases, as we show below, adding noise promotes transport. The additive force $f_{2}(t)$ are given by (2). All these magnitudes and parameters are in dimensionless form.

The current defined by Eq. (1) is time-shift invariant, i.e., it fulfils the symmetry (3) due to the dissipation. Therefore, if $v$ is a smooth functional such that its functional Taylor series exists, then Theorem 1 of [21] assures that

$$
v=\sum_{k=0}^{\infty}\left(\epsilon_{1}^{q_{2}} \epsilon_{2}^{q_{1}}\right)^{k} C_{k}\left(\epsilon_{1}, \epsilon_{2}\right) \cos \left[k q_{1} \phi+\theta_{k}\left(\epsilon_{1}, \epsilon_{2}\right)\right],
$$

with $\theta_{0}\left(\epsilon_{1}, \epsilon_{2}\right)=0$, and functions $C_{k}\left(\epsilon_{1}, \epsilon_{2}\right)$ and the phase lags $\theta_{k}\left(\epsilon_{1}, \epsilon_{2}\right)$ are even in each $\epsilon_{j}, j=1,2$. Notice that the symmetry (5) holds since exchanging $f_{2}$ with $-f_{2}$ is equivalent to replacing $x(t)$ with $-x(t)$ in (7). The statistical properties of the Gaussian white noise are the same under the inversion of $\xi(t)$ to $-\xi(t)$. Therefore, all $C_{k}$ with even $k$ are zero. With this restriction, the first two terms in (8), for $q_{1}=q_{2}=1$, read

$$
v=v_{1} \cos \left(\phi+\theta_{1}\right)+v_{2} \cos \left(3 \phi+\theta_{2}\right)+E_{10}\left(\epsilon_{1}, \epsilon_{2}\right),
$$

where $v_{1}=\epsilon_{1} \epsilon_{2} C_{1}\left(\epsilon_{1}, \epsilon_{2}\right), v_{2}=\left(\epsilon_{1} \epsilon_{2}\right)^{3} C_{2}\left(\epsilon_{1}, \epsilon_{2}\right), C_{1}$ and $\theta_{1}$ are polynomials up to order 6 in $\epsilon_{j}$, and $C_{2}$ and $\theta_{2}$ are linear in $\epsilon_{1}^{2}$ and $\epsilon_{2}^{2}$.

For $q_{1}=1$ and $q_{2}=2, v$ is given by

$v=v_{1} \cos \left(\phi+\theta_{1}\right)+v_{2} \cos \left(3 \phi+\theta_{2}\right)+E_{15}\left(\epsilon_{1}, \epsilon_{2}\right)$,

where $E_{15}$ contains terms of order 15 or higher in each $\epsilon_{j}$; $v_{1}=\epsilon_{1}^{2} \epsilon_{2} C_{1}\left(\epsilon_{1}, \epsilon_{2}\right), v_{2}=\left(\epsilon_{1}^{2} \epsilon_{2}\right)^{3} C_{2}\left(\epsilon_{1}, \epsilon_{2}\right), C_{1}$ and $\theta_{1}$ are even polynomials in $\epsilon_{1}$ and $\epsilon_{2}$ up to order 10 , and $C_{2}$ and $\theta_{2}$, are even polynomials in $\epsilon_{1}$ and $\epsilon_{2}$ up to order 4 . In both cases, $\left(q_{1}=\right.$ $q_{2}=1$ or $q_{1}=1$ and $q_{2}=2$ ) we have identified three main regimes, which depend on the amplitudes of the harmonics, namely:

(i) Small-amplitude regime. Only the first term in (9) and (10) dominates and $C_{1}$ and $\theta_{1}$ do not depend on the amplitudes. By fixing all the parameters of the system, $v$ is a sinusoidal function on $\phi$.

(ii) Intermediate amplitude regime. The second term in (9) and (10) can be neglected. However, in contrast to the previous case, $C_{1}$ and $\theta_{1}$ do depend on $\epsilon_{1}$ and $\epsilon_{2}$. Therefore, the current reversals can be achieved by modifying the amplitudes. The sinusoidal behavior of $v$ persists.

(iii) Large amplitude regime. The effect of the second term in (9) and (10) is observed, and therefore $v$ is no longer a sinusoidal function.

The previous analysis remains valid for the overdamped dynamics. To describe the overdamped system we set $m \rightarrow 0$ in Eq. (7):

$$
\alpha \dot{x}=-U^{\prime}(x)\left[1+f_{1}(t)\right]+f_{2}(t)+\sqrt{2 D} \xi(t) .
$$

Moreover, time reversal now implies that by changing $f_{1}(t) \rightarrow$ $f_{1}(-t), f_{2}(t) \rightarrow f_{2}(-t)$ and $x(t) \rightarrow \pi-x(-t)$, the Eq. (11) remains invariant and

$$
v\left[f_{1}(-t), f_{2}(-t)\right]=v\left[f_{1}(t), f_{2}(t)\right] .
$$

This symmetry fixes all the phase lags in Eq. (8) to zero. Therefore, all the phase lags in Eqs. (9) and (10) are also zero. Nevertheless, current reversals may still be observed by changing the amplitudes of the forces. For instance, in the intermediate regime, a variation in the parameters around the values for which $v_{1}\left(\epsilon_{1}, \epsilon_{2}\right)=0$ in Eqs. (9) and (10), could make $v$ change its sign.

In the following section, all these findings are verified by means of simulations of Eqs. (7) and (11).

\section{SIMULATIONS OF THE LANGEVIN EQUATION}

Simulations of the stochastic differential Eqs. (7) and (11) have been performed using the Heun method and the secondorder weak predictor-corrector method [25]. The final time of integration is 2000 , the time step is either 0.1 or 0.01 , and results are averaged over 10000 realizations unless specified otherwise in the figure caption.

The current $v$ is computed by means of

$$
v=\left\langle\frac{x\left(t_{f}\right)-x\left(t_{r}\right)}{t_{f}-t_{r}}\right\rangle
$$




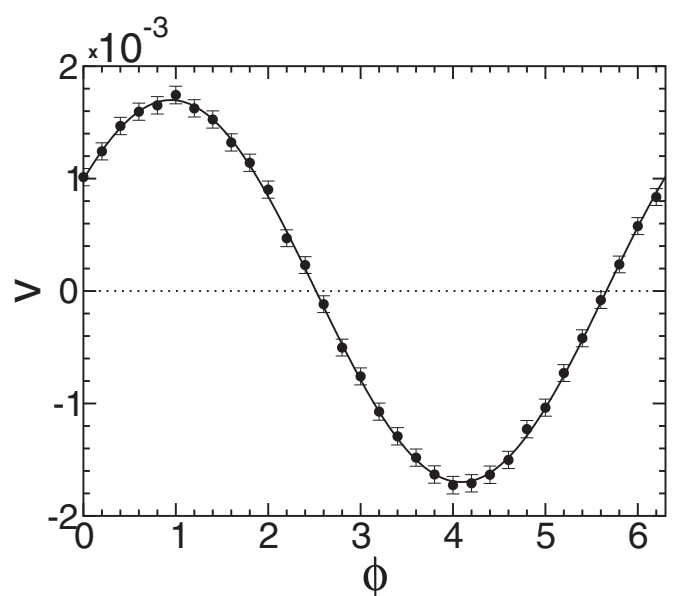

FIG. 1. $v$ vs $\phi$ from simulations of (7). Filled circles with error bars: current in steady state computed from the Eq. (13). Solid line represents the fitting curve of the circles, $v=-0.00170 \cos (\phi+$ 2.195). Parameters: $m=1, \alpha=1, U_{0}=5, \epsilon_{1}=\epsilon_{2}=0.5, \omega=1$, $q_{1}=1, q_{2}=2$, and $D=1$. Dotted line represents zero velocity.

where $t_{r}$ and $t_{f}$ are the final time of integration and the transient time, respectively (see Fig. 1).

The sinusoidal behavior of $v(\phi)$ is characteristic of the small and intermediate amplitude regimes. In Fig. 1, a sinusoidal behavior of $v$ is observed as a function of the phase. Close to $\phi \approx 2.5$ and $\phi \approx 5.7$, the velocity changes its sign and current reversals can appear by varying the phase and other parameters of the system that have an influence on the phase lag.

By further increasing the amplitudes, the average velocity deviates from purely sinusoidal behavior and sinusoids of higher frequencies appear in its expansion. Indeed, in Fig. 2, the results from simulations of Eq. (7) can be fitted perfectly with two harmonics. In Figs. 1 and 2, we notice that on replacing $\phi$ with $\phi+\pi$ (this is equivalent to replacing $f_{2}$ with $-f_{2}$ ), $v$ changes its sign. This means that the symmetry (5) is fulfilled.

By fixing all the parameters, except $\epsilon_{1}$ and $\epsilon_{2}$, which vary according to $\epsilon_{1}=A \epsilon, \epsilon_{2}=A(1-\epsilon)$, we verify that

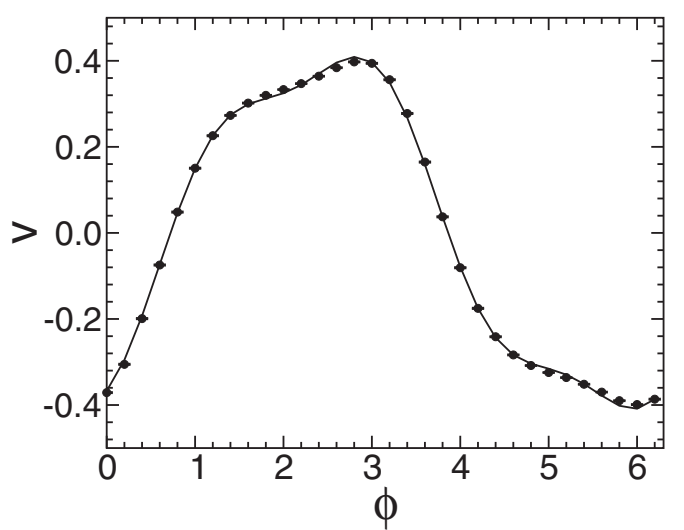

FIG. 2. $v$ vs $\phi$ from simulations of (7) shows nonsinusoidal behavior (filled circles with error bars). Solid line represents the fitting curve $v=-0.4168 \cos (\phi+0.7713)-0.0686 \cos (3 \phi-$ 0.0909). Parameters: $m=1, \alpha=1, U_{0}=5, \epsilon_{1}=\epsilon_{2}=2, \omega=1$, $q_{1}=1, q_{2}=2$, and $D=1$. Final time of integration 1000 .

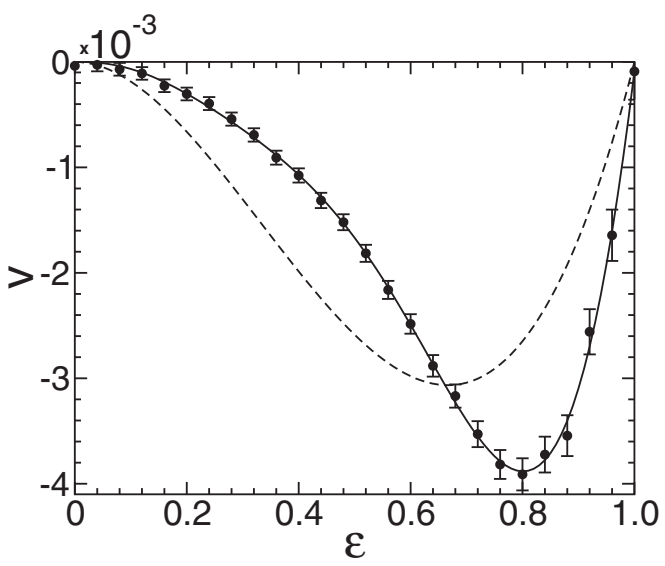

FIG. 3. $v$ vs $\epsilon$ from simulations of (7). Filled circles: current from the Eq. (13). Dashed line: fitting curve $v=-0.0207 \epsilon^{2}(1-\epsilon)$, predicted for small-amplitude limit. Solid line represents the fitting curve of the simulation points predicted in the intermediate amplitude regime, $v=\sum_{k=0}^{5} a_{k} \epsilon^{k+2}$, with $a_{0}=-0.002, a_{1}=-0.076, a_{2}=$ 0.367, $a_{3}=-0.747, a_{4}=0.664, a_{5}=-0.205$. Parameters: $m=1$, $\alpha=1, U_{0}=5, \epsilon_{1}=A \epsilon, \epsilon_{2}=A(1-\epsilon), A=1, \phi=4.09, \omega=1$, $q_{1}=1, q_{2}=2$, and $D=1$.

the dependence of $v$ on $\epsilon$ is different from the expected $v \sim \epsilon^{2}(1-\epsilon)$, which is valid for the small-amplitude regime (see Fig. 3).

In order to observe a current reversal via an amplitude change, first we fix all the parameters of Eq. (7) as in Fig. 1, except the amplitudes of the harmonics, which we have increased up to $\epsilon_{1}=\epsilon_{2}=1$. The amplitudes are now sufficiently large for the phase lags $\theta_{k}$ to be no longer constant and for them to depend on the amplitudes $\epsilon_{1}, \epsilon_{2}$ as in Eq. (8). We set a relative phase $\phi \approx 2.8$, which corresponds to an almost vanishing current for $\epsilon_{1}=\epsilon_{2}=1$ (not shown in the figures). A clear current reversal appears by modifying only the amplitudes around these values following $\epsilon_{1}=2 \epsilon$, $\epsilon_{2}=2(1-\epsilon)$ with $\epsilon \in[0,1]$, as shown in Fig. 4. The inversion

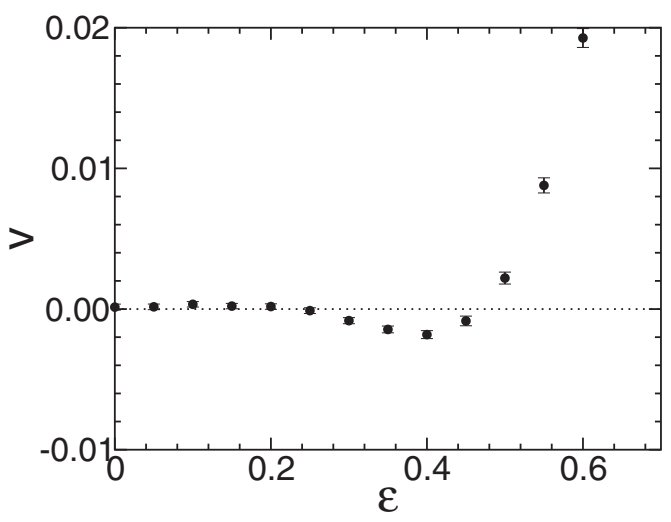

FIG. 4. $v$ vs $\epsilon$ from simulations of (7) shows that the direction of the current changes at approximately $\epsilon=0.5$. Parameters: $m=1$, $\alpha=1, U_{0}=5, \epsilon_{1}=2 \epsilon, \epsilon_{2}=2(1-\epsilon), \omega=1, \phi=2.8, q_{1}=1, q_{2}=$ 2 , and $D=1$. Final time of integration 1000 . Dotted line represents zero velocity. 


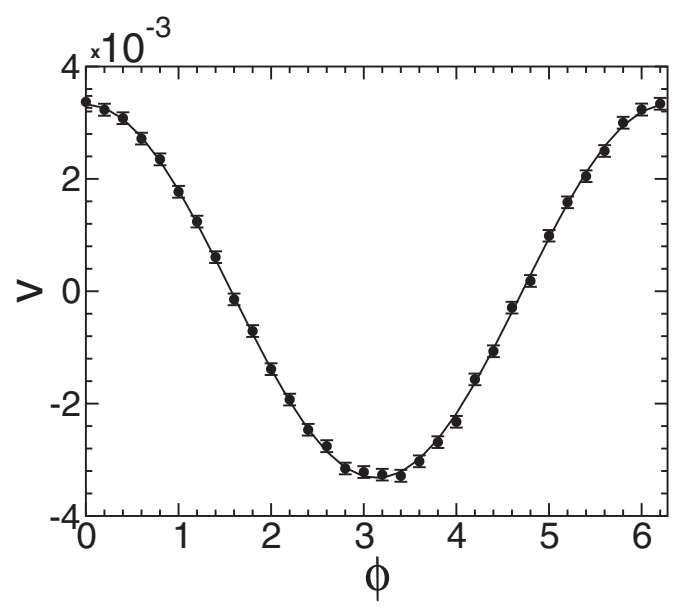

FIG. 5. $v$ vs $\phi$ from simulations of the overdamped system (11) (filled circles with error bars). Solid line represents the fitting curve $v=0.0033 \cos (\phi)$. Parameters: $\alpha=1, U_{0}=5, \epsilon_{1}=\epsilon_{2}=0.5, \omega=$ $1, q_{1}=1, q_{2}=2$, and $D=1$.

of the current occurs around $\epsilon=0.5$, which corresponds to values $\epsilon_{1}=\epsilon_{2}=1$ and a vanishing $v$, as expected.

\section{A. Overdamped dynamics of Brownian particle}

Interestingly, the maximum current shown in Fig. 5 for the overdamped particle is greater than the maximum current reached when the inertial term remains in the Langevin equation, see Fig. 1. Notice that the parameters in both figures are the same, except the inertial term which is omitted in the simulations reported in Fig. 5. This effect resembles the enhancement of the movement due to the dissipation studied in Refs. $[10,20]$ in the relativistic particle driven by a biharmonic force.

This striking phenomenon vanishes when the amplitudes are increased (the maxima of the currents shown in Figs. 2 and 6 are almost the same). On increasing the amplitudes, a small deviation from the sinusoidal behavior of $v$ as a function of the phase $\phi$ is also observed in the overdamped system, see Fig. 6.

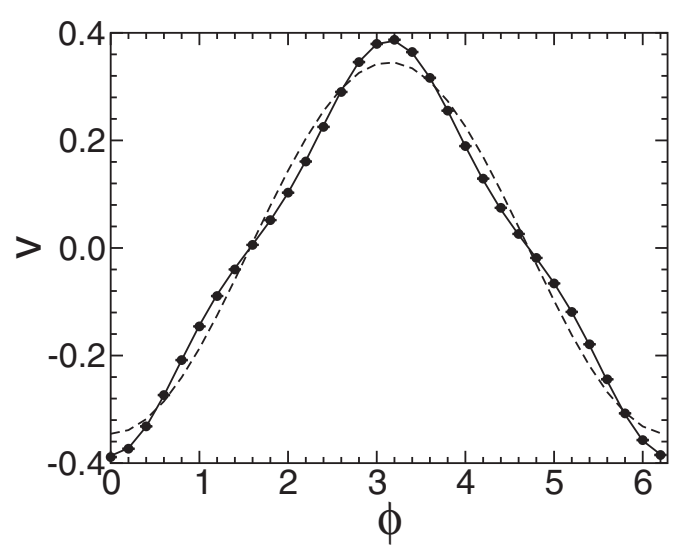

FIG. 6. $v$ vs $\phi$ from simulations of the overdamped system (11), filled circles with error bars. Dashed and solid lines are the fitting curves $v=-0.345 \cos (\phi)$ and $v=-0.344 \cos (\phi)-0.042 \cos (3 \phi)$, respectively. Parameters: $\alpha=1, U_{0}=5, \epsilon_{1}=\epsilon_{2}=2, \omega=1, q_{1}=1$, $q_{2}=2$, and $D=1$.

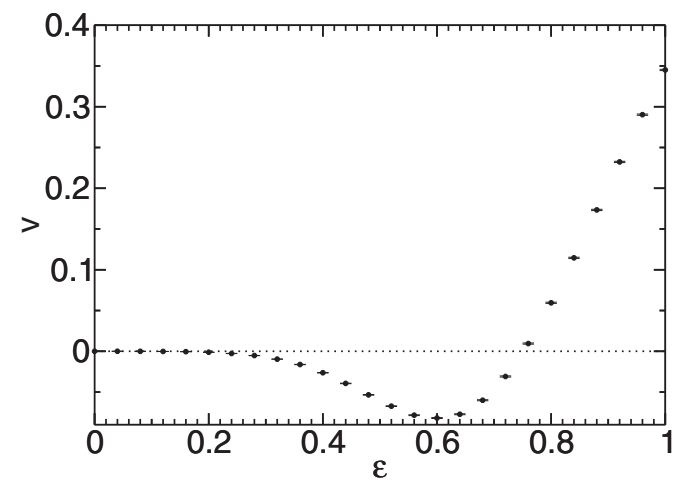

FIG. 7. $v$ vs $\epsilon$ from simulations of (11) shows that the direction of the current changes around $\epsilon \approx 0.75$. Amplitudes of $f_{1}$ and $f_{2}$ are varied simultaneously as $\epsilon_{1}=2 \epsilon, \epsilon_{2}=2 \epsilon$. The rest of parameters are: $\alpha=1, U_{0}=5, \omega=1, q_{1}=1, q_{2}=2, \phi=2.8$, and $D=1$. 20000 realizations.

In the overdamped dynamics, the phase lags are fixed to zero and the search for current reversals associated to changes in the amplitudes of the harmonics becomes a more difficult task. In order to observe a current reversal by changing the amplitudes of the forces, we must proceed in a different fashion. Results from simulations shown in Figs. 5 and 6 reveal that, by changing the amplitudes $\epsilon_{1}$ and $\epsilon_{2}$ from 0.5 to 2 when $\phi \approx 3$, the direction of motion can be inverted. Therefore, by setting the phase, for instance at $\phi=2.8$, and varying the amplitudes in the form of $\epsilon_{1}=2 \epsilon, \epsilon_{2}=\epsilon_{1}$ with $\epsilon \in[0,1]$, an inversion of the current is expected for a value of $\epsilon_{1}$ between 0.5 and 2. These results are shown in Fig. 7. Finally, Fig. 8 shows that an inversion is also observed when the amplitudes are modified while keeping the total amplitude $\epsilon_{1}+\epsilon_{2}$ constant. Moreover, Fig. 8 shows that $v=0$ when $\epsilon=0$ (no modulation of the potential) or $\epsilon=1$ (no additive force).

In Figs. 7 and 8, the inversion of the current occurs at $\epsilon_{1} \approx 1.5$. Indeed, by fixing all the parameters and changing $\epsilon_{1}$ and $\epsilon_{2}$, the contour plot (Fig. 9, left) shows that the current vanishes when $\epsilon_{1} \approx 1.5$. However, for other sets of parameters,

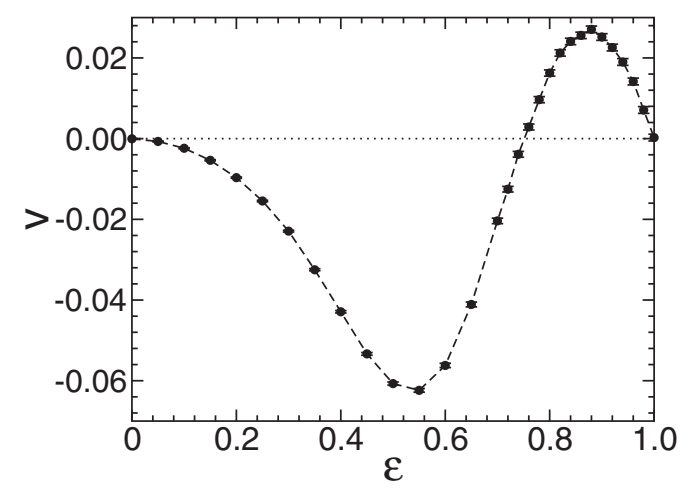

FIG. 8. $v$ vs $\epsilon$ from simulations of (11) shows that the direction of the current also changes around $\epsilon \approx 0.75$ (filled circles) when the amplitudes are varied with constant total amplitude 2 as $\epsilon_{1}=2 \epsilon$, $\epsilon_{2}=2(1-\epsilon)$. The rest of parameters are $\alpha=1, U_{0}=5, \omega=1$, $q_{1}=1, q_{2}=2, \phi=2.8$, and $D=1.20000$ realizations. 

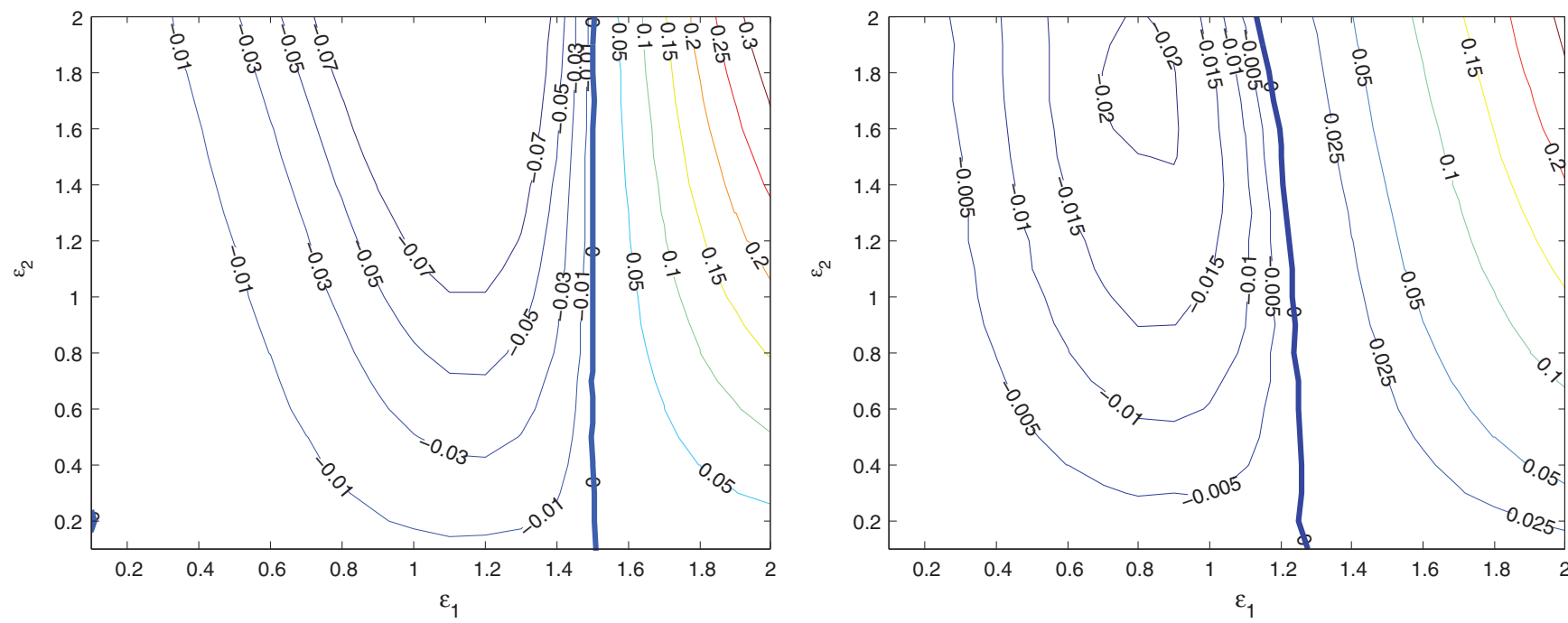

FIG. 9. (Color online) Contour plots $v$ as functions of $\epsilon_{1}$ and $\epsilon_{2}$ from simulations of (11). The thick blue line marks the inversion of the current. Left: $U_{0}=5$. Right: $U_{0}=2.5$. The rest of parameters are $\alpha=1, \omega=1, q_{1}=1, q_{2}=2, \phi=2.8$, and $D=1$.

for instance taking $U_{0}=2.5$ (see Fig. 9, right), the reversal current appears for different values of $\epsilon_{1}$.

\section{SUMMARY}

In this work, we study the dynamics of particles, driven by a harmonic force and subjected to white noise, when they are in a spatially symmetric potential that is modulated by a harmonic function. Both the applied force and the modulation of the potential are time symmetric and the current $v$ fulfils the symmetry (5). Dissipation is also included in the description; therefore the current is time-shift invariant and the theory developed in Ref. [21] can be applied.

We show that this theory predicts three different regimes for our system, which depend on the amplitudes of the two harmonics, namely: (i) A small-amplitude regime where the current, $v \sim \epsilon_{1}^{q_{2}} \epsilon_{2}^{q_{1}} \cos \left(\phi+\theta_{1}\right)$, is a sinusoidal function with a phase lag, $\theta_{1}$, independent of the amplitudes. This regime has been predicted by the collective coordinate theory and confirmed by simulations in the framework of soliton ratchets (see Ref. [19] and references therein). (ii) The intermediate amplitude regime, where $v$ is still a sinusoidal function, although $\theta_{1}$ is no longer a constant. This means that the sinusoidal behavior alone cannot guarantee that the amplitudes of the harmonics are small. Therefore, in addition to experiments on optical lattices reported in Ref. [18], in order to determine the regime where the system lies, it is necessary to investigate the dependence of $v$ on the amplitudes $\epsilon_{1}$ and $\epsilon_{2}$. Once the intermediate regime is reached, current reversals via an amplitude change is expected. This phenomenon is confirmed by simulations of the underdamped Langevin Eq. (7). It is worthy of note that current reversals have been found to be present in the overdamped limit, where the current satisfies the time-reversal symmetry (12). (iii) Large-amplitude regime, where we show that the nonsinusoidal behavior of the current, predicted by the theory, is due to the increasing strength of the two harmonics.

Apart from the results presented in Figs. 1-8, we have also performed simulations for all the set of parameters of Figs. 1-8, but we fixed the strength of the noise $D=0$. In all cases, the computed current is zero (of order of $10^{-9}$ or less). Therefore, for the set of parameters studied here the noise together with the action of the harmonics generate the transport.

Finally, it is pointed out that, according to the theory developed in Ref. [21], the main phenomena studied here using a specific model, can appear in other physical systems that satisfy the same symmetries, including experimental realizations in Josephson junctions [14,26,27] and optical lattices [15], in which a number of the above results have been reported. Other results, however, require verification through experiments.

\section{ACKNOWLEDGMENTS}

We acknowledge financial support through: Grants No. FIS2011-24540 (N.R.Q.) and FIS2011-22644 ENFASIS (L.D.); from Ministerio de Economía y Competitividad (Spain); Grants No. FQM207 (N.R.Q.), and No. P09-FQM4643 (N.R.Q.), from Junta de Andalucía (Spain); and especially a grant from the Alexander von Humboldt Foundation (Germany) through Research Fellowship for Experienced Researchers SPA 1146358 STP (N.R.Q.). Part of the calculations of this work were performed in the high capacity cluster for physics, funded in part by Universidad Complutense de Madrid, Spain and in part with Feder FUNDS. This is a contribution to the Campus of International Excellence of Moncloa, CEI Moncloa.
[1] A. Ajdari, D. Mukamel, L. Peliti, and J. Prost, J. Phys. I France 4, 1551 (1994).
[2] P. Hänggi and R. Bartussek, Lect. Notes Phys. 476, 294 (1996).

[3] R. D. Astumian, Science 276, 917 (1997). 
[4] P. Reimann, Phys. Rep. 361, 57 (2002).

[5] P. Hänggi and F. Marchesoni, Rev. Mod. Phys. 81, 387 (2009).

[6] H. Linke, Appl. Phys. A 75, 167 (2002).

[7] C. S. Lee, B. Jankó, I. Derényi, and A. L. Barabási, Nature (London) 400, 337 (1999).

[8] H. Linke, T. E. Humphrey, A. Lofgren, A. O. Sushkov, R. Newbury, R. P. Taylor, and P. Omling, Science 286, 2314 (1999).

[9] J. E. Villegas, S. Savel'ev, F. Nori, E. M. González, J. V. Anguita, R. García, and J. L. Vicent, Science 302, 1188 (2003).

[10] M. Salerno and Y. Zolotaryuk, Phys. Rev. E 65, 056603 (2002).

[11] F. Jülicher, A. Ajdari, and J. Prost, Rev. Mod. Phys. 69, 1269 (1997).

[12] S. Ooi, S. Savel'ev, M. B. Gaifullin, T. Mochiku, K. Hirata, and F. Nori, Phys. Rev. Lett. 99, 207003 (2007).

[13] E. R. Kay, D. A. Leigh, and F. Zerbetto, Angew. Chem. Int. 46, 72 (2007).

[14] A. V. Ustinov, C. Coqui, A. Kemp, Y. Zolotaryuk, and M. Salerno, Phys. Rev. Lett. 93, 087001 (2004).

[15] M. Schiavoni, L. Sánchez-Palencia, F. Renzoni, and G. Grynberg, Phys. Rev. Lett. 90, 094101 (2003).

[16] L. Dinis, E. González, J. Anguita, J. Parrondo, and J. Vicent, New J. Phys. 9, 366 (2007).
[17] P. Hänggi and R. Bartussek, in Current Topics in Physics (World Scientific, Singapore, 1998), Vol. 1, pp. 524-530.

[18] R. Gommers, V. Lebedev, M. Brown, and F. Renzoni, Phys. Rev. Lett. 100, 040603 (2008).

[19] E. Zamora-Sillero, N. R. Quintero, and F. G. Mertens, Phys. Rev. E 74, 046607 (2006).

[20] N. R. Quintero, J. A. Cuesta, and R. Alvarez-Nodarse, Phys. Rev. E 81, 030102 (2010).

[21] J. A. Cuesta, N. R. Quintero, and R. Alvarez-Nodarse, Phys. Rev. X 3, 041014 (2013).

[22] D. Cubero, V. Lebedev, and F. Renzoni, Phys. Rev. E 82, 041116 (2010).

[23] X. Noblin, R. Kofman, and F. Celestini, Phys. Rev. Lett. 102, 194504 (2009).

[24] L. Morales-Molina, F. G. Mertens, and A. Sánchez, Phys. Rev. E 72, 016612 (2005).

[25] P. E. Kloeden and E. Platen, Numerical Solution of Stochastic Differential Equations (Springer, Berlin, 1995).

[26] F. Falo, P. J. Martínez, J. J. Mazo, T. P. Orlando, K. Segall, and E. Trías, Appl. Phys. A 75, 263 (2002).

[27] M. Beck, E. Goldobin, M. Neuhaus, M. Siegel, R. Kleiner, and D. Koelle, Phys. Rev. Lett. 95, 090603 (2005). 